Revue d'histoire de l'Amérique française

ATS REVUE D.HISTOIRE DE L'AMÉRIQUE FRANÇAISE

\title{
Explorer la consommation dans une perspective historique
}

\section{Magda Fahrni}

Volume 58, numéro 4, printemps 2005

La consommation

URI : https://id.erudit.org/iderudit/012209ar

DOI : https://doi.org/10.7202/012209ar

Aller au sommaire du numéro

\section{Éditeur(s)}

Institut d'histoire de l'Amérique française

\section{ISSN}

0035-2357 (imprimé)

1492-1383 (numérique)

Découvrir la revue

\section{Citer cet article}

Fahrni, M. (2005). Explorer la consommation dans une perspective historique. Revue d'histoire de l'Amérique française, 58(4), 465-473.

https://doi.org/10.7202/012209ar d'utilisation que vous pouvez consulter en ligne.

https://apropos.erudit.org/fr/usagers/politique-dutilisation/ 


\section{Explorer la consommation dans une perspective historique}

MAGDA FAHRNI ${ }^{\mathrm{I}}$

Département d'histoire

Université du Québec à Montréal

Dourquoi étudier la consommation dans une perspective historique?
Comment l'examen de la consommation nous renseigne-t-il sur les spécificités d'une époque et d'un lieu? Quelle lumière fait-il sur les mœurs, le niveau de vie et les débats politiques présents dans une société donnée? Depuis une quinzaine d'années, à l'échelle internationale, les recherches sur la consommation se multiplient. Le Québec ne fait pas exception à cette règle, et nous trouvons dans ce numéro spécial de la Revue d'histoire de l'Amérique française quelques exemples de travaux récents dans le domaine.

La consommation, telle qu'elle est analysée dans les écrits d'historiens, a plusieurs définitions. Dans un premier temps, on l'interprète comme la consommation littérale d'aliments et de boissons ${ }^{2}$. Plus souvent, cependant, les historiens la définissent selon son sens «secondaire»: participer au marché et aux transactions commerciales, voire dépenser. Mais au-delà de sa simple définition, quelle est la signification de la consommation? Pour certains chercheurs, il s'agit surtout de comprendre la place des

1. Je remercie Christine Hudon pour ses précieux commentaires sur le fond et la forme de ce texte.

2. Voir, par exemple, Warren Belasco et Philip Scranton, dir., Food Nations: Selling Taste in Consumer Societies (New York, Routledge, 2002). 
pratiques consommatrices dans la sphère politique. La consommation engendre-t-elle une dépendance apolitique, voire non réfléchie, des consommateurs à l'égard des manufacturiers et des biens et du marché? Ou peut-on (devrait-on) considérer les manières de consommer et les choix des consommateurs comme une prise de position idéologique, et même comme un acte politique? Pour d'autres historiens, l'important est de comprendre les liens entre la consommation et la construction identitaire. Comment la consommation crée-t-elle des identités sociales - individuelles ou collectives? Comment peut-elle servir de stratégie de distinction sociale et même de stratégie d'exclusion? L'acte de consommer permet-il à l'acheteur individuel de s'épanouir? La consommation a-t-elle un potentiel libérateur?

La consommation est un thème qui se prête à l'étude de toutes les sociétés et de toutes les époques. Que l'on pense aux émeutes populaires provoquées par les crises de subsistance à l'ère préindustrielle, aux débats autour du "luxe» dans l'Europe du xviII siècle ou bien aux mouvements coloniaux de boycottage durant la Révolution américaine et les Rébellions bas-canadiennes de 1837-1838, il est clair que le concept de la consommation aide à comprendre la vie quotidienne à différentes périodes et à analyser les enjeux qui les traversent ${ }^{3}$. Cependant, la grande majorité des études récentes se penchent sur la période du "capitalisme consommateur» et de l'avènement d'une société de consommation dans la plupart des pays occidentaux au $\mathrm{xx}^{\mathrm{e}}$ siècle. Il s'agit d'une période où l'on assiste à la démocratisation de la consommation, et même à la jouissance plus répandue d'un certain luxe. Le développement de la production industrielle au $\mathrm{XIX}^{\mathrm{e}}$ siècle engendrait une consommation de masse et était, en même temps, stimulé par elle. Au cours du $\mathrm{xx}^{\mathrm{e}}$ siècle - époque de régimes fordistes, de salaires plus élevés, de vacances payées et d’États-providence structurés, du moins en partie, par la pensée keynésienne et donc par la reconnaissance officielle de la nécessité de la consommation - de nombreux citoyens européens et nord-américains ont pu se permettre, pour la première fois, d'acheter des biens au-delà du strict nécessaire ${ }^{4}$.

3. T. H. Breen, The Marketplace of Revolution: How Consumer Politics Shaped American Independence (New York/Oxford, Oxford University Press, 2004); Francis Back, «L'étoffe de la liberté: politique textile et comportements vestimentaires du mouvement patriote", Bulletin d'histoire politique, 10,2 (hiver 2002): 58-71; Allan Greer, «La république des hommes: les Patriotes de 1837 face aux femmes", Revue d'histoire de l'Amérique française, 44,4 (printemps 1991): 507-528; Maxine Berg et Elizabeth Eger, dir., Luxury in the Eighteenth Century: Debates, Desires and Delectable Goods (Houndmills, Balsingstoke, Hampshire, Palgrave, 2003).

4. Voir, par exemple, Kristin Ross, Aller plus vite, laver plus blanc : la culture française au tournant des années soixante (Paris, Abbeville, 1997). 
L'historiographie de la consommation touche à une multitude d'objets. Citons d'abord les études qui s'intéressent à la consommation des produits essentiels : aliments, logements, vêtements. Pendant longtemps, et peutêtre plus encore parmi les historiens britanniques et français, les chercheurs se sont intéressés à la question du «niveau de vie». L’industrialisation a-t-elle engendré une augmentation du niveau de vie des ouvriers? Le sort de la classe ouvrière s'est-il, au contraire, aggravé durant et après la Révolution industrielle $e^{5}$ ? Par ailleurs, et plus récemment, on observe chez certains historiens un intérêt pour la consommation de luxe - tissus précieux, bijoux, café, caviar ${ }^{6}$. Dans ces études, la consommation est intimement liée à la question du statut social et à la notion du «bon goût». L'article de Catherine Ferland, dans le présent numéro, qui porte sur l'élite de la Nouvelle-France et la consommation de l'alcool, trouve sa place dans cette historiographie. Celui de Caroline Coulombe également, puisqu'il traite du développement, tout au long $\mathrm{du} \mathrm{xx}^{\mathrm{e}}$ siècle, d'un intérêt pour la présentation esthétique des repas et pour des aliments «exotiques».

L'historiographie récente s'intéresse également aux lieux de la consommation - aux marchés publics, par exemple, et surtout aux grands magasins, en Europe comme en Amérique du Nord7. Pour ce qui est du Québec, nous n'avons qu’à penser aux travaux de Michelle Comeau sur Dupuis Frères, Eaton et Morgan, ou encore à ceux de Joanne Burgess sur les magasins-entrepôts du Vieux-Montréal ${ }^{8}$. Ces études s’intéressent à la

5. Par exemple, Maurice Halbwachs, La classe ouvrière et les niveaux de la vie: recherches sur la hiérarchie des besoins dans les sociétés industrielles contemporaines (Londres, Gordon and Breach, 1970); Arthur J. Taylor, dir., The Standard of Living in Britain in the Industrial Revolution (London, Methuen, 1975). Pour le Québec et le Canada, voir Terry Copp, Classe ouvrière et pauvreté: les conditions de vie des travailleurs montréalais 1897-1929 (Montréal, Boréal Express, 1978); Jean de Bonville, JeanBaptiste Gagnepetit: les travailleurs montréalais à la fin du XIX siècle (Montréal, L’Aurore, 1975); Eleanor Bartlett, «Real Wages and the Standard of Living in Vancouver, 1901-1929», BC Studies, 51 (1981): 3-62.

6. Maxine Berg et Helen Clifford, dir., Consumers and Luxury: Consumer Culture in Europe, 16501850 (Manchester, Manchester University Press, 1999). Voir également Jukka Gronow, Caviar with Champagne: Common Luxury and the Ideals of the Good Life in Stalin's Russia (Oxford/New York, Berg, 2003), recensé par Jarrett Rudy dans ce numéro.

7. Sean Gouglas, «Produce and Promotion: Covent Garden Market, the Socioeconomic Elite, and the Downtown Core in London, Ontario, 1843-1915", Urban History Review/Revue d'histoire urbaine, 25,1 (octobre 1996) : 3-18; Geoffrey Crossick et Serge Jaumain, dir., Cathedrals of Consumption: The European Department Store, 1850-1939 (Aldershot [England], Brookfield [Vermont], Ashgate, 1999); Susan Porter Benson, Counter Cultures: Saleswomen, Managers, and Customers in American Department Stores, 1890-1940 (Urbana, University of Illinois Press, 1986).

8. Michelle Comeau, «Les grands magasins de la rue Sainte-Catherine à Montréal: des lieux de modernisation, d'homogénéisation et de différenciation des modes de consommation ", Revue d'histoire de la culture matérielle, 41 (printemps 1995) : 58-68; Joanne Burgess, «Le centre victorien: 
consommation comme spectacle et à l'étalage des biens. Elles insistent sur l'importance du visuel, de l'esthétique. Elles nous montrent ces lieux de consommation comme lieux de modernité mettant en valeur de nouvelles inventions, telles les ascenseurs et l'éclairage artificiel. L'article de Lorraine O’Donnell, dans ce numéro, s'inscrit dans cette lignée. Les historiens qui se sont intéressés aux lieux de consommation ont souvent démontré un intérêt connexe pour l'histoire du marketing, des publicités et des stratégies de vente ${ }^{9}$.

Ces études des "cathédrales de la consommation» ont porté un intérêt particulier à la question du genre. Les grands magasins y sont analysés à la fois comme lieux de sociabilité féminine et de travail féminin. Ils sont perçus également comme lieux de rencontre des différentes classes sociales, notamment les vendeuses de la classe ouvrière et les acheteuses de la bourgeoisie. Pour certains chercheurs, les grands magasins, par le fait qu'ils offraient aux femmes la possibilité d'occuper l'espace public même quand elles étaient privées de droits politiques, avaient un potentiel émancipateur. De plus, la consommation offrait aux femmes la possibilité de se réaliser, car elle leur permettait de transformer leur identité - ou, du moins, leur apparence - en achetant. Les grands magasins ont été planifiés dans le but d'engendrer le désir, la tentation même, de consommer. Mais comme l'a remarqué l'historienne Elaine S. Abelson, cela a souvent produit des conséquences moins positives. Son livre sur les femmes et le vol à l'étalage démontre que ce délit, qui est devenu un phénomène important à la fin du $\mathrm{XIx}^{\mathrm{e}}$ siècle, était une réponse à ce même désir, cette même tentation, de consommer - et qu'il était donc partie intégrante de la culture de la consommation ${ }^{10}$.

À vrai dire, les consommateurs étudiés par les historiens sont souvent des consommatrices. Plus souvent qu'autrement, la bourse familiale se trouvait entre les mains des femmes mariées. Les ménagères, acheteuses

commerce et culture, 1850-1880 ", dans Gilles Lauzon et Madeleine Forget, dir., L'histoire du VieuxMontréal à travers son patrimoine (Sainte-Foy, Publications du Québec, 2004).

9. Stuart Ewen, Captains of Consciousness: Advertising and the Social Roots of the Consumer Culture (New York/Toronto, McGraw-Hill, 1976); David Monod, Store Wars: Shopkeepers and the Culture of Mass Marketing, 1890-1939 (Toronto, University of Toronto Press, 1996); Russell Johnston, Selling Themselves: The Emergence of Canadian Advertising (Toronto, University of Toronto Press, 2001).

10. Elaine S. Abelson, When Ladies Go A-Thieving: Middle-Class Shoplifters in the Victorian Department Store (New York, Oxford University Press, 1989), 6, 11. Pour d'autres analyses "genrées» des lieux de consommation, voir William R. Leach, «Transformations in a Culture of Consumption: Women and Department Stores, 1890-1925 ", Journal of American History, 71 (septembre 1984): 319-342; ainsi que les essais dans Victoria de Grazia et Ellen Furlough, dir., The Sex of Things: Gender and Consumption in Historical Perspective (Berkeley, University of California Press, 1996). 
des produits essentiels, sont donc des actrices importantes dans ces histoires de la consommation. Dans ces études, nous voyons des femmes qui fréquentaient les grands magasins, les marchés publics, les supermarchés et les épiceries du coin. Les historiens ont démontré que c'était habituellement les femmes qui élaboraient le budget familial, qui développaient des stratégies pour joindre les deux bouts, qui boycottaient certaines denrées essentielles et qui protestaient auprès des commerçants et des autorités politiques lorsqu'elles trouvaient les prix trop élevés ${ }^{11}$. Cela est si vrai qu'on a facilement tendance à accepter la dichotomie "production masculine, consommation féminine». Mais il ne faut pas oublier que, pour de nombreuses femmes mariées, assurer et gérer la consommation familiale faisaient partie de leur travail "productif ${ }^{12}$ ». En fait, pour la plupart des femmes, la consommation était du travail, non rémunéré dans le cas des femmes mariées, rémunéré pour celles qui travaillaient dans le secteur des services. La consommation appartenait à la fois à la sphère privée et à la sphère publique: pour les historiens, elle peut servir de lien entre l'une et l'autre ${ }^{13}$.

Un autre courant important de l'historiographie qui nous intéresse est celui qui traite du rôle de l'État dans la consommation. Les politiques économiques officielles déterminent, dans une mesure importante, les possibilités de consommation, notamment en temps de crise économique et en temps de guerre. Ainsi, certains historiens des États-Unis se sont penchés sur les rapports entre le New Deal du gouvernement Roosevelt des années 1930 et les citoyens - définis dorénavant comme citoyensconsommateurs ${ }^{14}$. Les politiques de consommation des gouvernants en temps de guerre - rationnement, contrôles sur les prix, les loyers, et les salaires - ont retenu l'attention des historiens du Canada, des États-Unis, de la Grande-Bretagne et des pays de l'Europe occidentale. L'article d'Yves

11. Bettina Bradbury, Familles ouvrières à Montréal. Âge, genre et survie quotidienne pendant la phase d'industrialisation (Montréal, Boréal, 1995); Denyse Baillargeon, Ménagères au temps de la Crise (Montréal, Éditions du remue-ménage, 1991); Magda Fahrni, "Counting the Costs of Living: Gender, Citizenship, and a Politics of Prices in 1940s Montreal ", Canadian Historical Review, 83,4 (décembre 2002): 483-504.

12. Joy Parr, Domestic Goods: The Material, the Moral, and the Economic in the Postwar Years (Toronto, University of Toronto Press, 1999), 6.

13. Jacqueline K. Dirks, "Of Women and Things», Journal of Women's History, 16,1 (2004): 173.

14. Lizabeth Cohen, "The New Deal State and the Making of Citizen Consumers", dans Susan Strasser et al., dir., Getting and Spending: European and American Consumer Societies in the Twentieth Century (Washington, D.C., Cambridge University Press/German Historical Institute, 1998); Meg Jacobs, "”Democracy”s Third Estate“: New Deal Politics and the Construction of a "Consuming Public" ", International Labor and Working-Class History, 55 (printemps 1999) : 27-51. 
Tremblay, dans ce numéro, s'inscrit directement dans cette tendance. Il souligne l'énorme pouvoir de gérer l'économie qu'avait le gouvernement fédéral durant la Deuxième Guerre mondiale. Dans les contextes canadien et britannique, ce nouveau pouvoir était jumelé, chez certains fonctionnaires, à un intérêt croissant pour les politiques keynésiennes. Ces dernières présumaient une consommation importante de la part des citoyens - et faisaient, en fait, de tous les citoyens des consommateurs.

Si l'État est un acteur primordial dans la consommation, on ne doit pas présumer que les citoyens ne faisaient qu'attendre ses décisions. Les citoyens-consommateurs, les historiens l'ont démontré, ont non seulement réagi aux directives étatiques, mais ils ont formulé leurs propres demandes afin d'accéder à ce qu'ils considéraient être un niveau de vie raisonnable ${ }^{15}$. Ils ont constitué les Ligues des consommateurs et des consommatrices et y ont participé. Au Québec, ces ligues ont été particulièrement actives à la fin de chacune des grandes guerres du $\mathrm{xx}^{\mathrm{e}}$ siècle. Cette participation citoyenne à la formulation de la politique consommatrice et économique est en fait un élément manquant dans les essais qui suivent - à d'autres chercheurs de suivre cette piste prometteuse!

Catherine Ferland nous livre une exploration originale et détaillée de la consommation de boissons alcoolisées chez l'élite de la Nouvelle-France au XVIII ${ }^{\mathrm{e}}$ siècle. Les vins chers, un marqueur du "bon goût», servaient à dessiner des distinctions de classe et de "race» entre, d'un côté, l'élite canadienne, et de l'autre côté, «le peuple» et «les Sauvages». Si la consommation des boissons alcoolisées chères était une technique d'exclusion sociale, la consommation des vins français, en particulier, servait en même temps à rapprocher l'élite canadienne de l'élite française, à réduire l'écart métaphorique entre la colonie et la métropole. Par ailleurs, Ferland démontre que la consommation d'alcool faisait appel à tout un ensemble d'accessoires (vaisselle, bouteilles, glace) et de pratiques (le toast, le service) qui servaient à construire l'identité de l'élite canadienne et à distinguer cette élite du reste de la population. L'auteure rappelle que le statut social n'était pas la seule caractéristique qui définissait quand, où et comment on consommait l'alcool en Nouvelle-France: ici, le genre et l'âge étaient, eux aussi, des facteurs déterminants.

15. James Hinton, «Militant Housewives: The British Housewives' League and the Attlee Government », History Workshop Journal, 38 (1994): 128-156; Julie Guard, «Women Worth Watching: Radical Housewives in Cold War Canada », dans Gary Kinsman et al., dir., Whose National Security? Canadian State Surveillance and the Creation of Enemies (Toronto, Between the Lines, 2000). 
Caroline Coulombe s'intéresse également à la consommation à la fois dans le sens d'ingestion et dans celui de participation au marché. Elle se sert du livre de cuisine comme "texte» révélateur de grandes tendances sociales au Québec, du début de l'époque industrielle jusqu'à la Révolution tranquille. Consciente que le livre de cuisine consiste surtout en modèles diététiques et en "prescriptions adressées aux ménagères», Coulombe suggère néanmoins qu'il puisse servir de source afin de comprendre les habitudes de consommation et les "nouveaux comportements alimentaires». Les comportements alimentaires $\mathrm{du} \mathrm{xx}^{\mathrm{e}}$ siècle étaient façonnés par des considérations économiques, religieuses et scientifiques, par la croissance d'une "industrie alimentaire» et par le développement de la cuisine standardisée. Ils étaient également accompagnés de nouveaux rôles sociaux liés à la consommation et à la production domestique. La "parfaite hôtesse" des années 1950 et 1960, affirme Coulombe, était celle qui privilégiait la créativité, la nouveauté et la gastronomie. Dans ces années de salaires plus élevés, d'économies familiales plus stables, d'ouverture sur le monde extérieur et de valorisation intense de la mère au foyer, les repas n'étaient plus une simple question de subsistance, ni uniquement de nutrition. L'esthétique était importante: la salle à manger était devenue la scène, le repas, un spectacle, et la femme mariée, le personnage principal.

Cet intérêt pour les dimensions visuelles de la consommation est partagé par Lorraine O’Donnell dans son article sur le magasin Eaton de Montréal. L'aspect de la consommation privilégié dans cet article est la mode féminine, et surtout la façon dont la mode, telle qu'elle était promue par Eaton, donnait aux femmes montréalaises l'occasion de "voyager», ne serait-ce que de façon «virtuelle», à l'étranger. Plus souvent qu'autrement, la haute couture, pour Eaton et pour ses clientes, voulait dire Paris. Au milieu du $\mathrm{xx}^{\mathrm{e}}$ siècle, les vêtements importés de France par les acheteurs d'Eaton (dont Doreen Day, figure emblématique chez Eaton), et ensuite vendus aux clientes montréalaises, ainsi que les défilés de mode faisaient connaître à celles-ci le dernier chic parisien. Dans une société de plus en plus ouverte sur le monde, il y avait ici une manière d'aller chercher des éléments «étrangers» et même "exotiques»... même si on n'avait pas les moyens de voyager réellement. Ainsi, propose O’Donnell, la mode européenne était en quelque sorte "libératrice» pour les consommatrices québécoises. En même temps, ces "voyages virtuels" n'étaient pas accessibles à toutes les Montréalaises. Le grand magasin Eaton occupait une place particulière dans le paysage économique et culturel de la ville. S’il n'était pas le magasin «chic anglophone» qu'était 
Morgan, il n'était pas non plus le "magasin du peuple», et surtout du peuple canadien-français, qu'était Dupuis Frères ${ }^{16}$.

La contribution d'Yves Tremblay à ce numéro spécial met l'accent sur un acteur moins présent dans les trois premiers articles: l'État. Durant la Deuxième Guerre mondiale, l'État fédéral s'est donné la responsabilité, et le pouvoir, de diriger la consommation du pays. En temps de guerre, il fallait éviter une possible inflation. Afin de subvenir aux besoins des soldats et des pays alliés outre-mer, il fallait également composer avec la pénurie de certains biens et denrées au pays. Ottawa a donc imposé des plafonds sur les prix et sur les salaires et, à l'instar des autres pays des forces alliées, a instauré un système de rationnement d'un certain nombre de denrées, dont le sucre, le beurre, le thé, le café, la viande et l'essence. Somme toute, comme le titre de l'article de Tremblay l'indique, le rôle d'Ottawa était de «brider» la consommation pour la durée de la guerre et de s'assurer que les citoyens respectent ses nombreux règlements. Au Québec et au Canada, les désirs individuels affrontaient les besoins collectifs, et la frontière entre la "nécessité» et le «luxe» devint plus rigide. L'existence du marché noir démontre que ce n'était pas tous les citoyens qui se conformaient aux règlements fédéraux. Elle soulève des questions quant au patriotisme de certains citoyens, et elle suggère la volonté continue des citoyens de consommer (même des biens considérés comme du «luxe») durant la guerre. Qui plus est, le pacte tacite entre gouvernants et gouvernés semble n'avoir existé que durant les hostilités militaires. Comme l'indique l'échec du rationnement de la viande en septembre 1945, les restrictions furent moins bien supportées après la victoire. Les contrôles économiques imposés par le gouvernement fédéral demandaient donc tout un travail de surveillance : il fallait "mesurer» l'opinion publique au moyen de sondages et convaincre la population, par le biais de la propagande, de se conformer aux multiples directives étatiques.

Jarrett Rudy clôt ce numéro special de la Revue d'histoire de l'Amérique française par une note critique qui évalue six livres récents dans l'histoire internationale de la consommation. Son analyse de ces ouvrages, qui traitent du Canada, des États-Unis, de la Grande-Bretagne, de la France, de l'Union soviétique et de l'Amérique latine, démontre que les rapports de pouvoir et les questions identitaires demeurent au cœur de ces études très diverses. Rudy se penche notamment sur leurs différentes manières d'aborder la dimension politique de la consommation, que ce soit par le

16. Michelle Comeau, «Les grands magasins de la rue Sainte-Catherine», 66. 
biais des discours, des idéologies, des politiques étatiques ou bien des mouvements de consommateurs. De plus, il porte son attention sur la façon dont la classe sociale, le genre et l'ethnie (ou la "race») ont influé sur la consommation dans les différentes sociétés à l'étude. Enfin, Rudy explore la place de la nation comme catégorie d'analyse dans ces ouvrages et il se demande si l'État-nation est en fait le meilleur angle sous lequel aborder le phénomène consommateur dans le passé.

Que reste-t-il donc à faire dans l'histoire de la consommation? Quels sont les questionnements à poursuivre et à développer dans le contexte de l'histoire de l'Amérique française? Y a-t-il des leçons à tirer de l'historiographie actuelle?

Nous croyons d'abord qu'il est essentiel de ne pas faire abstraction de la dimension politique dans l'étude de la consommation. Le "magasinage » peut nous révéler les intérêts, voire les prises de position idéologiques des individus, des ménages et des nations. De nos jours, l'intérêt croissant pour le "consumérisme éthique» est preuve de la prise de conscience récente de la nature politique de nos achats ${ }^{17}$. En même temps - et ceci est, à notre avis, la deuxième "morale » de cette histoire -, il ne faut pas non plus surestimer le potentiel émancipateur de la consommation. Les contraintes imposées par la production et par les salaires demeurent importantes et limitent de manière fondamentale les possibilités de consommer. Ces possibilités varient beaucoup d'une société à une autre et au sein même d'une société donnée. Les choix des manufacturiers et des gouvernants pèsent parfois plus lourd que ceux des acheteurs-citoyens. Et la surconsommation est en train de générer d'immenses problèmes environnementaux ${ }^{18}$. Somme toute, il est fort possible que l'historien américain Jackson Lears ait raison lorsqu'il suggère que le bonheur promis par la consommation est plus une question de plaisir individuel que de bien collectif ${ }^{19}$.

17. Laure Waridel, Acheter, c'est voter. Le cas du café (Montréal, Éditions Écosociété, 2005).

18. The Worldwatch Institute, Linda Starke, dir., State of the World 2004. Special Focus: The Consumer Society (New York, W. W. Norton \& Company, 2004).

19. Jackson Lears, "Two on the Aisle», Radical History Review, 37 (1987): 79-80. 